\title{
Construcción de aprendizaje en el aula: una propuesta pedagógica que emerge del análisis del programa de televisión infantil Guillermina y Candelario*
}

\section{Construction of learning in the classroom: a pedagogic proposal that emerge from the children's show Guillermina and Candelario}

\author{
Carlos Gutiérrez-González \\ Universidad del Norte, Colombia. \\ gutierrezgonzalez@uninorte.edu.co
}

\begin{abstract}
RESUMEN
Este artículo analiza si la serie de televisión infantil Guillermina y Candelario promueve en su audiencia la alfabetización de valores y el respeto por la naturaleza y si fomenta la estrategia del Edu-Entretenimiento en los niños. Para alcanzar estos objetivos, se planteó una investigación cualitativa donde se realizaron cinco grupos focales con infantes, cuatro entrevistas en profundidad con profesores de básica primaria y un análisis de contenido cualitativo de cuatro episodios. De la información analizada emerge un modelo de construcción del aprendizaje en el aula, fundamentado sobre la construcción de sentido propiciado por el proceso dialógico entre el educador y el educando. Se concluye que, con este programa de televisión, se podrían diseñar estrategias pedagógicas que complementen la falta de información en aspectos ecológicos, sociales y éticos, se persuada a los infantes a tener un cambio positivo en su comportamiento individual y se eduquen sobre las normas sociales.
\end{abstract}

Palabras claves: educación, edu-entretenimiento, método de enseñanza, televisión, valores sociales.

\section{ABSTRACT}

This article identifies if the children's show Guillermina and Candelario promotes in its audience the values literacy and the respect for nature and if it encourages the Edu-Entertainment strategy in children. To reach this aims, it was proposed a qualitative investigation where they were made five children's focus groups, four in-deep interviews with primary school professors and a qualitative content analysis of four chapters. Of the analysed information emerges a construction model of learning in the classroom, based on the construction of meaning propitiated by the dialogic process between the educator and the pupil. It is concluded that, with the children's television show, it is possible to design pedagogic strategies that complement the lack of information in ecological, social and ethical aspects, persuade the infants to have a positive change in their individual behaviour and educate them about the social norms.

Key words: education, edu-entertainment, educational methods, television, social values.

* Este artículo es resultado del proceso de la formación doctoral en Comunicación de la Universidad del Norte, Colombia, el cual se financió con recursos del Fondo Nacional de Financiamiento para la Ciencia, la Tecnología e Innovación FcTEI Del Sistema General de Regalías (SGR). 


\section{INTRODUCCIÓN}

Guillermina y Candelario (Rincón, Rincón \& de Jesús, 2010) es una serie de televisión infantil que inicialmente fue emitida por el canal regional Telepacífico y luego por Señal Colombia, un canal público con cobertura nacional. El programa es dirigido a niñas y niños entre los 5 y 8 años de edad y es inspirado en los escenarios del pacífico colombiano (Fosfenos Media, n.d.). Su objetivo es recrear la cotidianidad de Guillermina y Candelario, un par de hermanos afrodescendientes, "quienes con su curiosidad y deseo de exploración convierten cada día en una aventura llena de conocimiento y diversión [...] inspirando respeto por la naturaleza y los seres que la habitan" (RTVC, 2016). En la historia, los infantes suelen estar acompañados por su abuelo, un personaje que se dedica a la pesca, actividad económica tradicional de esa región.

En la actualidad, se han emitido cuatro temporadas para un total de 71 capítulos. En la primera, cada episodio tuvo una duración promedio de 4 minutos, en la segunda de 22 y en la tercera y la cuarta de 12. Esta última temporada incluye temas como la muerte, la identidad, la sexualidad y el racismo, con el fin de profundizar en temáticas que despiertan el interés de los niños, quienes a su edad, empiezan a cuestionarse acerca de todo lo que les rodea (Señal Colombia, 2017). La historia de Guillermina y Candelario se ha expandido a otras plataformas digitales, por ejemplo, cuenta con 10 clips de 2 minutos cada uno publicados en el canal de YouTube de Señal Colombia, Mi Señal ${ }^{1}$, y un sitio $w_{e} b^{2}$ donde los infantes pueden encontrar "manualidades, actividades, historias y juegos relacionados con el Pacífico colombiano" (MaguaRed, 2015). Además, la serie ha sido emitida en Chile, Perú, Venezuela y fue doblada al portugués para emitirse en TV Brasil con el título Guilhermina y Candelario (ComKids, 2015).

Asimismo, este proyecto audiovisual ha sido selección oficial en el Prix Jeunesse Iberoamericano y nominado a otros festivales internacionales como Animamundi, Divercine y Festicine Kids. Para Marcela Rincón, directora y guionista de la serie, ha sido gratificante producir cada uno de los episodios, especialmente, cuando reciben llamadas de niños de toda Colombia y les escriben diciendo que les encantan los personajes. Igualmente, la directora comenta que

las profesoras están trabajando con nuestros capítulos en las escuelas, es decir, los niños están aprendiendo con Guillermina y Candelario y están aprendiendo del Pacífico. La serie está siendo una herramienta pedagógica muy poderosa en muchos lugares del país y muchas profesoras lo están utilizando en las aulas del colegio. Entonces, esto nos parece encantador (Entrevista personal al periodista Jairo Aristizábal, junio 20 de 2017) $)^{3}$.

Bajo este contexto, el portal educativo "Colombia Aprende" del Ministerio de Educación Nacional, incluye al programa infantil dentro de la franja que Señal Colombia ofrece a esta audiencia con contenidos de "alta calidad y una buena dosis de

\footnotetext{
Tomado de: https://www.youtube.com/watch?v=g6hBR0-p_GI\&list=PL07RPVp258LDa5c3xnQzowoOuDH08W9p0

Tomado de: http://www.guillerminaycandelario.com/

Tomada de: https://www.youtube.com/watch?v=7Spu8GJiRIk
} 
eduentretenimiento" (Rivera, 2017). En ese sentido, los investigadores académicos se han interesado por el Edu-Entretenimiento desde varios frentes temáticos, por ejemplo, indagando por el codiseño y el trabajo colaborativo en proyectos de realidad aumentada entre investigadores e infantes (Alhumaidan, Pui, Lo \& Selby, 2018), por la atención de los niños respecto a las actividades que practican en el aula con la tecnología como mediadora del proceso de aprendizaje (Barreto, Vasconcelos \& Orey, 2017), por la frecuencia de los términos y mensajes educativos emitidos por canales como Cartoon Network, Disney y Nickelodeon (Hunting et al., 2018), por las motivaciones de los videojuegos educativos que integran el aprendizaje y la experiencia del juego (Egenfeldt-Nielsen, 2006) y por la representación de los mensajes morales explícitos o implícitos para la construcción de valores (Feng, 2016).

Para el caso de este artículo, las preguntas que se pretenden responder son: ¿cómo el fomenta el eduentretenimiento el programa de televisión infantil Guillermina y Candelario en su audiencia?, y la siguiente, sustentada en las propuestas de investigación de EduEntretenimiento planteadas por Jarvin (2015) es, ¿cómo se puede implementar el contenido este programa en las intervenciones en el aula?

\section{EL EDU-ENTRETENIMIENTO}

La trayectoria del Edu-Entretenimiento (E-E) como estrategia de comunicación que promueve el cambio social ha sido larga, productiva y exitosa, donde se han beneficiado el sector agrícola, la planificación familiar, la salud pública, los derechos humanos, el medio ambiente y la educación (Tufte, 2015). Singhal y Rogers (2002) definen a la educación de entretenimiento como el

proceso de diseñar e implementar deliberadamente un mensaje mediático para entretener y educar, con el fin de aumentar el conocimiento de los miembros de la audiencia sobre un tema educativo, crear actitudes favorables, cambiar las normas sociales y cambiar el comportamiento (p. 117).

En esa perspectiva, la educación para el entretenimiento definida como la ubicación intencional del contenido educativo en los mensajes de entretenimiento, ha recibido especial atención de los académicos en las últimas décadas, principalmente, evaluando los efectos de las intervenciones en la audiencia (Singhal \& Rogers, 2002; Tufte, 2015). Sin embargo, Singhal y Rogers (2002) proponen cinco agendas desde las cuales se pueden ubicar las investigaciones alrededor del Edu-Entretenimiento:

- Agenda 1: Las investigaciones teóricas de la educación para el entretenimiento deberían prestar mayor atención a la variabilidad entre las intervenciones de E-E.

- Agenda 2: Las investigaciones teóricas de E-E deberían prestar más atención a las diversas resistencias a las intervenciones de E-E.

- Agenda 3: la teorización de E-E se beneficiará de investigaciones minuciosas de los aspectos retóricos, lúdicos y afectivos de E-E.

- Agenda 4: La investigación de "efectos" de E-E debería considerar el empleo de una comprensión más amplia de los cambios individuales, grupales y de nivel social. 
Estudios Pedagógicos XLV, $\mathrm{N}^{\circ}$ 3: 127-143, 2019

CONSTRUCCIÓN DE APRENDIZAJE EN EL AULA: UNA PROPUESTA PEDAGÓGICA QUE EMERGE DEL ANÁLISIS DEL PROGRAMA DE TELEVISIÓN INFANTIL GUILLERMINA Y CANDELARIO

- Agenda 5: La investigación de "efectos" de E-E debería ser más receptiva al pluralismo metodológico y al ingenio de medición. (p. 120)

Por su parte Tufte (2015) establece tres generaciones que se han presentado en el campo del Edu-Entretenimiento. La primera generación centra su problema en la falta de información, su noción de entretenimiento se plantea desde una herramienta para transmitir un mensaje y un agente de cambio apunta a un grupo objetivo pasivo. Su noción de cambio, a través de una pedagogía de la persuasión, está dirigida al comportamiento individual y a educar sobre las normas sociales. En consecuencia, el resultado esperado sería el cambio en normas y comportamiento individual.

La segunda generación, además de apuntar a resolver problemas de información, también incluye los conflictos de las desigualdades estructurales. Su noción de entretenimiento es un género dinámico y su noción de cambio está en el comportamiento individual, las normas sociales y las condiciones estructurales. El resultado esperado, además del cambio de comportamiento individual, consiste en la generación de debates públicos y privados en torno al problema tratado.

La tercera generación trata problemas de desigualdades estructurales, relaciones de poder y conflicto social. Su noción de entretenimiento se centra en un proceso de cultura popular y se plantea el género como forma de expresión, mientras que la noción de cambio, además de las particulares de las generaciones anteriores, incluye el cambio de las relaciones de poder. Los resultados esperados buscan la articulación del proceso social y político, un cambio estructural y una acción colectiva.

Para el caso de esta investigación, el foco está en la Agenda 3 y en la primera generación, profundizando en las estrategias narrativas y lúdicas que emplean los creadores de Guillermina y Candelario. Por esto, se sigue la recomendación de Singhal y Rogers (2002) cuando manifiestan que las investigaciones teóricas de Edu-Entretenimiento deberían tomar el papel de las emociones más en serio, centrándose en la comunicación del afecto, los sentimientos y las emociones de los personajes de los programas con los miembros de la audiencia.

\subsection{LA RELACIÓN DEL CURRÍCULO CON LOS CONTENIDOS AUDIOVISUALES INFANTILES}

Desde la escuela, los profesores se han preocupado por generar estrategias lúdicas que les faciliten a sus estudiantes comprender de una manera práctica los núcleos temáticos abordados en el aula. En ese sentido, la tecnología brinda herramientas que proporcionan nuevas formas de aprender, dinámicas y agradables, en especial, para los estudiantes de preescolar y básica primaria. Una de las formas más efectivas para el proceso de aprendizaje de los infantes es haciendo uso de la imagen. Al respecto, Perceval y Tejedor (2008) manifiestan que el poder de la imagen se puede utilizar en el aula, siempre y cuando el educador tenga en consideración que la capacidad de atención de los estudiantes ha disminuido y la velocidad del lenguaje audiovisual ha aumentado ya que el ritmo lento no favorece la atención del alumno. De allí la importancia de elegir bien los contenidos.

Lo anterior, también permite mejorar la comunicación del docente con el estudiante, siempre y cuando el primero esté interesado en apoyar sus prácticas pedagógicas en las herramientas tecnológicas y que estas sean del gusto de ambos actores y los contenidos 
atiendan las necesidades de la comunidad académica (De Souza \& do Amaral, 2008). Asimismo, estos recursos deben estar contextualizados para determinar la importancia situacional y su significado, en caso contrario, el público objetivo necesitaría contextualizarse para permitir que se produzca un sentido apropiado de la información (Hill \& Hannafin, 2001; Tufte, 2001), reconociendo la capacidad de la audiencia para interpretar el mensaje y del docente para emplearlo en el momento más apropiado.

En consecuencia, "el diálogo entre el lenguaje audiovisual y el lenguaje curricular debe ser dinámico y constante, el acuerdo entre el objetivo educativo y el soporte elegido también" (Perceval \& Tejedor, 2008, p. 160), por tal razón en este escenario, es prioritario conectar las dimensiones personales de los participantes con el proceso educativo. Una manera efectiva de hacerlo es a través de los contenidos producidos para la televisión donde la audiencia conecta sus "problemas, sus sentimientos y pasiones; y lo hacen con tal fuerza que llegan al mundo interior del espectador despertando pensamientos, valoraciones, cambios de actitud y percepción de la realidad" (Fabbro \& Sánchez-Labella, 2016, p. 12).

En este propósito, Vera (2011, citada por, Fabbro \& Sánchez-Labella, 2016) propone "usar los medios para construir conocimiento y difundir valores igualitarios, siendo en este punto donde se conjuga la función crítica y se vertebra el conocimiento y la experiencia" (p. 12). Para ilustrar esto, los dibujos animados se presentan como

un recurso fácil y accesible, prácticamente para toda la población infantil, para transmitir una serie de valores culturales y educativos que, paralelamente, se representan también a través de otros medios de comunicación. [...] Las narraciones televisivas animadas se consideran discursos que transmiten explícita o implícitamente los valores socialmente predominantes, que no siempre coinciden con los valores deseables desde el punto de vista educativo (Fabbro \& Sánchez-Labella, 2016, p. 17).

Por lo anterior, una de las funciones del rol social del maestro es orientar a sus educandos respecto a lo que observan y escuchan en los medios de comunicación masivos, por lo tanto, y como ya se ha mencionado, elegir de manera pertinente los contenidos a trabajar en el aula es uno de los requerimientos esenciales para que la dialéctica en la codificación y decodificación del mensaje tenga sentido y el objetivo de aprendizaje planteado en el currículo se cumpla. De esta manera, a través de la interacción es posible distinguir formas y prácticas propias en las que se negocia lo visto, lo apropiado y lo entendido (Zabala, 2016).

Los niños ven televisión en la época más determinante de su formación física, desarrollo mental y creación de hábitos y actitudes, por lo tanto, los creadores de contenidos infantiles buscan que su público se conecte con la trama (La Ferrara, 2016) y despierte admiración hacia el protagonista, quien cumple un rol específico en la historia y representa valores que transmiten una carga afectiva y emotiva significativa en la audiencia. Las características del personaje ídolo, en ocasiones, son imitadas por los infantes, por ejemplo, la vestimenta, los modos de conductas o los valores (Fabbro \& Sánchez-Labella, 2016).

Precisamente los valores y los antivalores tienen un papel importante en esta investigación, por tal motivo, se toman como ejemplo los empleados por Alvarez Rodríguez (2007), quien los presenta de acuerdo con la siguiente taxonomía: 
Tabla 1. Valores y antivalores

\begin{tabular}{|c|c|c|}
\hline Contenido & Valores & Antivalores \\
\hline Valores Corporales & $\begin{array}{l}\text { El alimento, la salud, el descanso, } \\
\text { la higiene. }\end{array}$ & $\begin{array}{l}\text { El hambre, la enfermedad, la } \\
\text { embriaguez, la obesidad. }\end{array}$ \\
\hline Valores Intelectuales & $\begin{array}{l}\text { Leer, escribir, estudiar, aprender, } \\
\text { enseñar, pensar, la crítica, la } \\
\text { creatividad, la reflexión. }\end{array}$ & $\begin{array}{l}\text { La ignorancia, el analfabetismo, } \\
\text { el adoctrinamiento. }\end{array}$ \\
\hline Valores Afectivos & $\begin{array}{l}\text { El sentimiento, la emoción, la } \\
\text { pasión, el amor, la ternura, la } \\
\text { sensibilidad, la afectividad. }\end{array}$ & $\begin{array}{l}\text { La tristeza, el dolor, el temor, el } \\
\text { miedo, la melancolía, el odio. }\end{array}$ \\
\hline Valores Estéticos & $\begin{array}{l}\text { El arte, la música, la pintura, la } \\
\text { escultura, la poesía, el teatro, la } \\
\text { literatura. }\end{array}$ & $\begin{array}{l}\text { Lo feo, lo desagradable, lo } \\
\text { antiestético. }\end{array}$ \\
\hline Valores Individuales & $\begin{array}{l}\text { La conciencia, la autonomía, la } \\
\text { personalidad, la individualidad, } \\
\text { el carácter, la singularidad, la } \\
\text { originalidad, la independencia, la } \\
\text { autosuperación, la autocrítica. }\end{array}$ & $\begin{array}{l}\text { La masificación, la alienación, el } \\
\text { egocentrismo. }\end{array}$ \\
\hline Valores Morales & $\begin{array}{l}\text { La bondad, la verdad, la virtud, } \\
\text { la pureza, la generosidad, la } \\
\text { justicia, la honradez, la igualdad, } \\
\text { la solidaridad, la voluntad, la } \\
\text { fidelidad, la dignidad. }\end{array}$ & $\begin{array}{l}\text { El robo, la mentira, la injusticia, la } \\
\text { corrupción, la violencia. }\end{array}$ \\
\hline Valores Sociales & $\begin{array}{l}\text { La familia, la fiesta, la política, } \\
\text { la comunidad, la democracia, } \\
\text { el bien común, el pacifismo, la } \\
\text { convivencia, la multiculturalidad, } \\
\text { el pluralismo político, la diversidad } \\
\text { cultural. }\end{array}$ & $\begin{array}{l}\text { La enemistad, la guerra, la } \\
\text { discordia, el individualismo. }\end{array}$ \\
\hline Valores Ecológicos & $\begin{array}{l}\text { El río, el campo, el agua, el aire } \\
\text { limpio, la flor, la montaña, la } \\
\text { luz, la naturaleza, la ecología, el } \\
\text { desarrollo sostenible. }\end{array}$ & $\begin{array}{l}\text { La contaminación, la } \\
\text { deforestación, los desechos } \\
\text { tóxicos, el armamento nuclear. }\end{array}$ \\
\hline Valores Instrumentales & $\begin{array}{l}\text { El dinero, el sueldo, la vivienda, } \\
\text { el coche, el teléfono móvil, el } \\
\text { vestido, los ordenadores, las } \\
\text { nuevas tecnologías. }\end{array}$ & $\begin{array}{l}\text { El consumismo, la miseria, el } \\
\text { chabolismo. }\end{array}$ \\
\hline Valores Religiosos & $\begin{array}{l}\text { Dios, Jesucristo, el evangelio, } \\
\text { la oración, la misa, la iglesia, la } \\
\text { mezquita, el Corán. }\end{array}$ & La increencia, el materialismo. \\
\hline
\end{tabular}

Fuente. Elaboración propia a partir de Alvarez Rodríguez (2007). 
Esta clasificación permite "obtener una medida aproximada de los valores de una persona o grupo y saber cómo se organizan y jerarquizan sus respectivas escalas axiológicas" (Alvarez Rodríguez, 2007, p. 157). De esta forma, el educador propicia situaciones desde sus currículos para abordar estrategias de formación integral dirigidas a sus estudiantes. Con base en lo anterior y siguiendo la recomendación de López Neira (2017) "de seguir desarrollando investigación que permita aumentar la disponibilidad de modelos efectivos de integración y uso de soluciones digitales en ambientes formales e informales de educación" (p. 101), el objetivo de este estudio es identificar si el programa infantil Guillermina y Candelario promueve en su audiencia la alfabetización de valores y el respeto por la naturaleza y si contribuye a fomentar la estrategia del Edu-Entretenimiento en los niños.

\section{MÉTODO}

Abordando un enfoque cualitativo, se realizaron cinco grupos focales con la participación de infantes entre los 4 y los 12 años de edad. Ellos fueron distribuidos de acuerdo con la clasificación de Del Río (2000): dos grupos con niños y niñas entre 4 y 7 años de edad, quienes prefieren los contenidos argumentales, por lo que, en esta etapa, tienen la capacidad de diferenciar entre los contenidos reales y ficticios; dos grupos con niños y niñas de 7 y 8 años, que tienen una comprensión débil y sus recuerdos son episódicos; y un grupo con menores entre los 9 y los 12 años, los cuales van perdiendo interés por la televisión, seleccionando mucho más los gustos de los programas que desean ver y centrando su atención en los elementos sonoros y visuales más relevantes para ellos.

Además, los menores seleccionados son residentes de áreas urbanas y rurales, estudiantes de escuelas públicas y con comportamientos heterogéneos en el aula de clase, por lo tanto, se acudió a una estrategia de muestreo de casos típicos (Lindlof \& Taylor, 2002). Luego, se diseñó una guía orientadora la cual fue validada por un experto en el tema y otro en el diseño metodológico. En este instrumento, se establecieron tres momentos.

En el primero, se habló del tema de la televisión y se les preguntó a los niños y niñas, entre otras cosas, por los contenidos que les gusta ver y si creen que estos les dejan enseñanzas; para el segundo, se inició con la proyección de un capítulo de Guillermina y Candelario (Temporada 1, Episodio: La lleva, Duración: 4') y se indagó por la historia, los personajes y el aprendizaje del capítulo que vieron; finalmente, se les mostró un segundo episodio ${ }^{4}$ (Temporada 3, Episodio: El anticuario de los mochileros, Duración: 12' o Temporada 3, Episodio: Una tarea muy divertida, Duración: 12'), donde además de realizar las mismas preguntas, se indagó si les gustaría que los profesores incluyeran estos contenidos en clase. Cada grupo focal tuvo una duración entre 60 y 80 minutos. Al finalizar, se les brindó un refrigerio a los niños y sus padres o adultos responsables firmaron los consentimientos informados para llevar a cabo el estudio.

De otro lado, se hicieron cuatro entrevistas a profesores de básica primaria que orientan las asignaturas de ética y ciencias naturales, áreas en las cuales hacen énfasis las creadoras del programa infantil. En el cuestionario, se dividió la entrevista en dos momentos, siguiendo la

Cuando la conexión a internet era la adecuada, se proyectó El anticuario de los mochileros, de lo contrario, se visualizaba Una tarea muy divertida. 
estrategia empleada en los grupos focales. En primera instancia, se abordaron las estrategias pedagógicas empleadas por el docente en el aula, la pertinencia de la televisión respecto con la educación y el conocimiento que tienen del Edu-Entretenimiento. La segunda parte inició con la visualización del capítulo La lleva, con el fin de tener testimonios del mismo episodio tanto de los infantes como de los adultos. Finalmente, se indagó por la posible implementación del contenido en el aula. Cada entrevista tuvo una duración entre 40 y 60 minutos y fueron realizadas en las instituciones educativas donde laboran.

Por último, se realizó un análisis de contenido aplicando la ficha implementada por Fabbro y Sánchez-Labella (2016) en su investigación. Allí, se consideraron los tipos de valores y contravalores representados en los tres capítulos proyectados a los sujetos de estudio, se identifican los personajes que los ejecuta y el tiempo de presencia en la pantalla. Así, con la triangulación de las tres técnicas seleccionadas, se acude a una de las estrategias de confiabilidad con la información recogida y se aplica la saturación como estrategia de validez interna.

\section{RESULTADOS}

Una vez se realizó el análisis de la información recogida en cada instrumento implementado en el trabajo de campo, resultaron las siguientes categorías: la educación y tecnología, en la que se encuentran las estrategias y herramientas pedagógicas implementadas en el aula, cuya importancia radica en las enseñanzas que resultan del diálogo generado entre la decodificación que realizan los niños de los contenidos visualizados y las preguntas que ellos hacen a los profesores; el aporte de Guillermina y Candelario a la educación, donde se define el rol de la televisión, los gustos en los contenidos infantiles y el contenido planteado en los episodios; y los valores en el mundo narrativo de Guillermina y Candelario, que se plantean desde los enunciados por Alvarez Rodríguez (2007). Como resultado del análisis de estas categorías, se propone un modelo para la construcción del aprendizaje en el aula a partir de la estrategia de Edu-Entretenimiento.

\subsection{LA EDUCACIÓN Y LA TECNOLOGÍA}

La evolución de la tecnología es un fenómeno que ha impactado la cotidianidad de la humanidad y la educación no es una excepción. Cada organización y cada individuo se han venido adaptando a los retos que exige la actualidad. En el caso de la educación, a directivos y docentes se les presentan desafíos generacionales porque deben pensar en las necesidades de aprendizaje de los niños y adolescentes que van ingresando y creciendo en las instituciones educativas. Así, algunos educadores han venido apoyándose en la tecnología para diseñar planes curriculares, microcurriculares y estrategias pedagógicas que contribuyan a que las clases en el aula sean más dinámicas, divertidas y entretenidas.

En ese sentido, los profesores que fueron entrevistados coincidieron en la importancia de implementar estrategias pedagógicas fundamentadas en la tecnología, por ejemplo, para el profesor Giovanny Ceballos, la tecnología y los contenidos que pueden enseñarse a través de una pantalla son herramientas valiosas para potenciar el aprendizaje, ya que

pueden generar variabilidad y otra posibilidad de aprendizaje. Debemos tener en cuenta que no todos los niños aprenden de la misma manera, forma y al mismo tiempo, 
entonces estas herramientas se pueden articular para que generen las posibilidades de que algunos niños adquieran unos conocimientos que, desde la parte tecnológica, puedan ser mucho más fáciles de adquirir (Entrevista personal, marzo 17 de 2018).

Sin olvidar algunas estrategias tradicionales como el aprendizaje a través del juego, la incorporación de la tecnología en el aula debe ser un complemento pedagógico que no sustituya el aporte del maestro. Al respecto, el profesor Jorge Cardona comenta que los contenidos audiovisuales y la música tienen la ventaja de que gustan a los estudiantes, ya que ellos

están acostumbrados a estar en la clase magistral, estar con sus guías, estar con su profesor que dicta y habla y ellos a veces como que lo miran a uno con aburrimiento. Entonces, ese tipo de cosas rompen lo convencional y es algo diferente. Así, ellos ponen atención, porque es más fácil estar viendo y escuchando que, por ejemplo, leyendo un texto. Esto es más sencillo para ellos y es más atractivo (Entrevista personal, febrero 15 de 2018).

No obstante, el diálogo cumple un papel relevante en ese complemento de la mediación tecnológica en la enseñanza y se convierte en otro componente importante en la formación del infante. Para esto, la profesora Olga Lucía Alzate considera fundamental la participación del estudiante, a través de actividades lúdicas "que lo involucren y lo incentiven a pensar, interpretar, argumentar, a trabajar las competencias básicas que son la base de la formación" . Asimismo, se evidenció en los grupos focales que los estudiantes siempre buscaban al docente para sentirse partícipe y activo en la actividad, propiciando un proceso dialógico con su maestro.

Por ejemplo, en el episodio La lleva (01'12”), el pájaro y la torta no querían jugar a la lleva con el tiburón. Uno de los niños pregunta: “¿cómo es que todo el mundo le tiene miedo al tiburón?", a lo que uno de los niños responde, "ay profe, ellos le tienen miedo al tiburón porque él se come a los demás animales y es muy peligroso". Más adelante (02'13"), una niña comenta que el tiburón "tiene una bandera blanca” y pregunta: ¿para qué, profe?", a lo que otra compañera responde, "está pidiendo la paz, no se va a comer a nadie, ¿cierto profe? Él no se come a nadie porque es mentira".

Lo anterior, es resultado de la decodificación del mensaje que hacen los niños del contenido observado. Este proceso de decodificación resulta importante en el aprendizaje del menor puesto que le permite asociar imágenes y sonidos con experiencias previas que derivan en la construcción de ideas y conocimientos. Por tal motivo, el profesor Ceballos, cuando habla del trabajo solidario, es porque

los niños, a partir del trabajo del uno con el otro, se fortalecen, generan nuevos aprendizajes a sus propios ritmos y de acuerdo con sus capacidades. Entonces, mi metodología es más una metodología activa que lleva al niño a que en todo momento esté creando, construyendo, proponiendo desde el ambiente propio donde el niño actúa (Entrevista personal, marzo 17 de 2018).

Entrevista personal, Olga Lucía Alzate, marzo 31 de 2018. 
Estudios Pedagógicos XLV, N 3: 127-143, 2019

CONSTRUCCIÓN DE APRENDIZAJE EN EL AULA: UNA PROPUESTA PEDAGÓGICA QUE EMERGE DEL ANÁLISIS DEL PROGRAMA DE TELEVISIÓN INFANTIL GUILLERMINA Y CANDELARIO

Esa construcción de sentido del mensaje les deja enseñanzas a los menores. En el caso de la actividad de los grupos focales, los infantes reconocieron algunas lecciones derivadas de las historias observadas, por ejemplo, "ayudar a las personas. Hacer las tareas bonitas. No pelear y si uno le pega al otro, pedir perdón. No discriminar a nadie y jugar con todos. Aceptarnos. Cuidar la naturaleza porque es muy bonita. Compartir lo que sabemos porque hay niños que no son capaces de hacer algunas cosas solitos. No rendirse. No tener miedo y perdonar".

De esta forma, se evidencia que el aprendizaje es una construcción permanente, situación fundamental para generar una motivación en los niños donde lo que van a aprender "les va a servir para la vida, lo va a hacer mucho mejor y le servirá en cualquier ambiente o escenario donde esté"'.

\subsection{EL APORTE DE GUILLERMINA Y CANDELARIO A LA EDUCACIÓN}

La discusión en la cual se presenta la disyuntiva de que la televisión influye de manera positiva o negativa al televidente, especialmente, al público infantil, está vigente y los profesores, con sus testimonios lo corroboran. Para ellos, es importante que los menores estén acompañados para que comprendan mejor el significado del mensaje,

porque son pocos los niños que eligen un programa que les guste o que les llegue a ellos de acuerdo con la edad. Por ejemplo, yo escucho a los niños decir que les encantan esas novelas de medianoche en las cuales hay violencia y contenidos sexuales que muchas veces el niño no ha identificado en sí, lo cual les trae incógnitas, generando preguntas muy profundas en el tema (Entrevista personal, Gladys Londoño, marzo 19 de 2018).

Esta consecuencia es generada, usualmente, porque "el papá o la mamá debe trabajar y no puede estar pendiente cien por ciento de su hijo. Al dejarlo solo, no hay un control de lo que puedan ver en la televisión" . En cambio, la televisión puede ser "un espacio de entretenimiento y aprendizaje, pero en programas adecuados y en compañía de la familia"8, "un espacio que propone aprendizajes, que capacita, que da la oportunidad de asimilar conceptos y aprendizajes" .

Por lo tanto, teniendo como aliada a la televisión, sus contenidos y la proactividad del educador, el Edu-Entretenimiento como estrategia comunicativa implementada en el aula, cobra valor para motivar al niño y despertar su interés por el aprendizaje a partir de las propuestas metodológicas que el educador genere. Para alcanzar estos logros, una alternativa sería articular los contenidos curriculares, con los audiovisuales y los gustos de los niños y niñas teniendo en cuenta sus rangos de edad. Por ejemplo, el grupo focal de 9 a 12 años, estuvo distraído y, por momentos, adoptando una conducta inadecuada durante la actividad ya que optaron por la indisciplina. El argumento que dieron al indagar por su comportamiento es que la narrativa no era de su interés, como sí lo son las telenovelas como La rosa de Guadalupe o la emisión de un partido de fútbol.

\footnotetext{
6 Entrevista personal, Giovanny Ceballos, marzo 17 de 2018.

7 Entrevista personal, Jorge Cardona, febrero 15 de 2018.

8 Entrevista personal, Gladys Londoño, marzo 19 de 2018.

9 Entrevista personal, Giovanny Ceballos, marzo 17 de 2018
} 
Respecto a los gustos televisivos de los integrantes de los grupos de 4 a 7 años, los menores mencionaron que los programas de "muñequitos, las películas de humor y de súper héroes o las películas de princesas" son sus favoritos. Para ellos, lo más importante es que los contenidos los entretengan y les enseñen cosas como bailar y cantar, además, que incluyan personajes atractivos, música y colores. Por lo tanto, ellos estuvieron más atentos a los capítulos de Guillermina y Candelario, ya que la música y los personajes fueron de su agrado y se sintieron identificados con las situaciones representadas por los protagonistas.

La música en Guillermina y Candelario es un elemento narrativo de primer orden y las interpretaciones corren por cuenta de los dos niños protagonistas y sus abuelos, especialmente, quienes lo hacen cuando comparten un sentimiento de felicidad o de enseñanza. Por ejemplo, la canción que más les gustó a los niños se presentó en el capítulo Una tarea muy divertida (10’50”). La lírica es la siguiente:

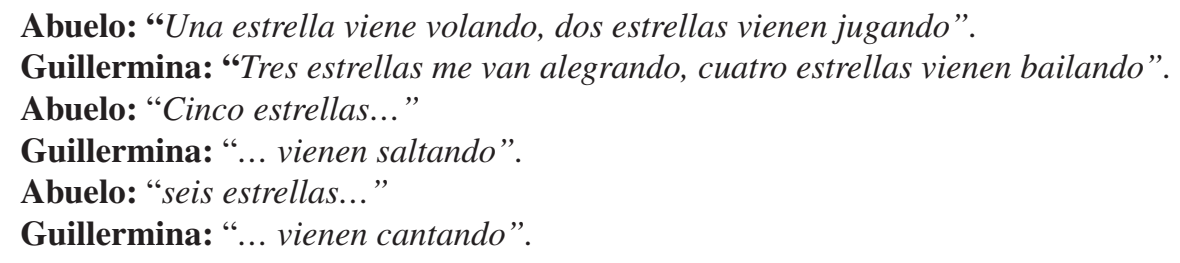

Abuelo: "Una estrella viene volando, dos estrellas vienen jugando".

Guillermina: "Tres estrellas me van alegrando, cuatro estrellas vienen bailando".

Abuelo: "Cinco estrellas..."

Guillermina: “... vienen saltando”.

Abuelo: "seis estrellas..."

Guillermina: “... vienen cantando".

Mientras la canción se escuchaba, los niños intentaron seguir el canto con la suma de las estrellas, mueven sus manos y su cuerpo y algunos manifiestan el gusto por el ritmo musical: “¡Ay!, qué canción más bonita”, "me voy a aprender la canción”. Asimismo, se abordó el tema de la identificación de los personajes, en este caso, porque "ellos son niños y nosotros también", porque "juegan y se divierten", "montan bicicleta", hasta uno de los menores manifestó que Candelario se parece a su "hermanito" por el color de la piel. Finalmente, una de las niñas, viendo el capítulo El anticuario de los mochileros, se muestra disgustada por algo que le ocurrió a Guillermina. La protagonista le saca la lengua a su abuelo y a Candelario porque el primero le dijo, al verle la cara sucia, que si la selva la había revolcado. Esto ocurrió porque Guillermina se cayó en un par de ocasiones mientras aprendía a montar bicicleta.

Para concluir este apartado, vale la pena resaltar que, para los profesores entrevistados es valioso que los estudiantes conozcan las culturas de otras regiones que no corresponden a las que ellos y los menores habitan, porque ven en Guillermina y Candelario, "un contenido muy práctico, donde cualquier niño, de cualquier región de nuestro país, puede asimilar, tranquilamente el mensaje que se quiere transmitir" ${ }^{\prime \prime}$. Además, el hecho de que los personajes sean "afrodescendientes, muestra inclusión. De pronto, los estudiantes no se van a sentir identificados al principio, pero se van a dar cuenta de que esa región del pacífico también pertenece a Colombia y van a conocer la diversidad de lo que es Colombia" ${ }^{11}$. Por lo anterior, "es necesario retomar otras culturas, otras formas de pensar, otros contextos, ya que Colombia es un país diverso. Así, aprovechamos de todo lo que nos brinda Colombia para aprender a quererla y a respetarla"12.

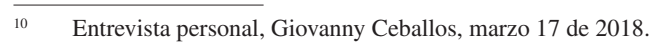

11 Entrevista personal, Jorge Cardona, febrero 15 de 2018.

12 Entrevista personal, Gladys Londoño, marzo 19 de 2018.
} 


\subsection{LOS VALORES Y ANTIVALORES EN EL MUNDO NARRATIVO DE GUILLERMINA Y CANDELARIO}

Una de las preguntas realizadas a los profesores fue, ¿cuáles son sus temas favoritos, los que usted no deja por fuera en sus asignaturas?, y la respuesta de los cuatro tuvo uno en común: los valores. Al respecto, ellos mencionan que, enseñando los valores como el respeto por la palabra, por el otro, por la naturaleza y el medio ambiente, los infantes tendrán una formación más elocuente en su proyecto de vida, formando personas integrales. De tal manera, los creadores de Guillermina y Candelario, consideraron algunos valores y contravalores para el desarrollo narrativo de sus historias.

Los valores intelectuales, como el carácter, la conciencia, la creatividad, el aprendizaje, la enseñanza, el estudio, el pensamiento y la reflexión; así como los valores morales, como la fidelidad, la generosidad, la justicia y la voluntad, son los que más se reiteran a lo largo de los cuatro episodios, los cuales están representados, especialmente, por Guillermina y Candelario. En cuanto a los contravalores se encuentran el egocentrismo, el miedo, la ignorancia y el enojo.

De acuerdo con lo anterior, el profesor Ceballos, después de ver el capítulo La lluvia, comenta la importancia que tiene este contenido porque

hace mucha referencia a lo que es el contacto de las personas con la naturaleza, el valor que se tiene por la naturaleza y lo importante de mantener las tradiciones, en este caso, dándole la bienvenida a la lluvia, resaltando que es para conservar el desarrollo sociocultural de la comunidad donde habitan Guillermina y Candelario (Entrevista personal, marzo 17 de 2018).

En ese sentido, otros valores ecológicos que se presentaron fueron la ecología, el río y el mar. En los valores sociales siempre se observó la familia como epicentro de unidad para resolver los problemas generados por el hambre, el dolor corporal y, especialmente, por el miedo, por ejemplo, cuando Guillermina tuvo una pesadilla donde se escondía de unos números que la perseguían en un bosque (Episodio: Una tarea muy divertida, 04'30").

Para cerrar, a los profesores les parece atractiva la idea de incluir los programas de Guillermina y Candelario dentro de sus estrategias pedagógicas, porque "de una forma motivante, se puede llevar al estudiante para que aprenda, ya que cuando se emplean varias estrategias, el niño se va a ver más motivado a aprender"'13. Al respecto, el profesor Ceballos comenta que

al paso que veía el capítulo, me imaginaba proponiendo clases para mis niños. Pienso que tienen una duración de tiempo que es acorde y los contenidos son muy pertinentes para el desarrollo de propuestas en las cuales nuestros niños desarrollen habilidades y se fomenten valores (Entrevista personal, marzo 17 de 2018).

Finalmente, el profesor Cardona señala que los infantes "se enseñan, desde pequeños, a cuidar su contexto. Nuestra misión es fortalecer los valores para poder llegar a decir que estamos formando una personita íntegra" ${ }^{14}$, aunque para lograr lo anterior, es importante

13 Entrevista personal, Gladys Londoño, marzo 19 de 2018.

14 Entrevista personal, Olga Lucía Alzate, marzo 31 de 2018. 
“conocer más el programa, para ver qué tipo de contenidos se tratan en los demás capítulos"15.

\subsection{MODELO DE CONSTRUCCIÓN DEL APRENDIZAJE EN EL AULA}

Con el análisis de la información realizado y desde un enfoque sociocultural que contempla la actividad o la estrategia pedagógica como unidad de análisis como lo plantea EgenfeldtNielsen (2006), se propone un modelo de construcción del aprendizaje en el aula a partir del Edu-Entretenimiento, como se aprecia a continuación:

Ilustración 1. Modelo de construcción del aprendizaje en el aula a partir del Edu-Entretenimiento

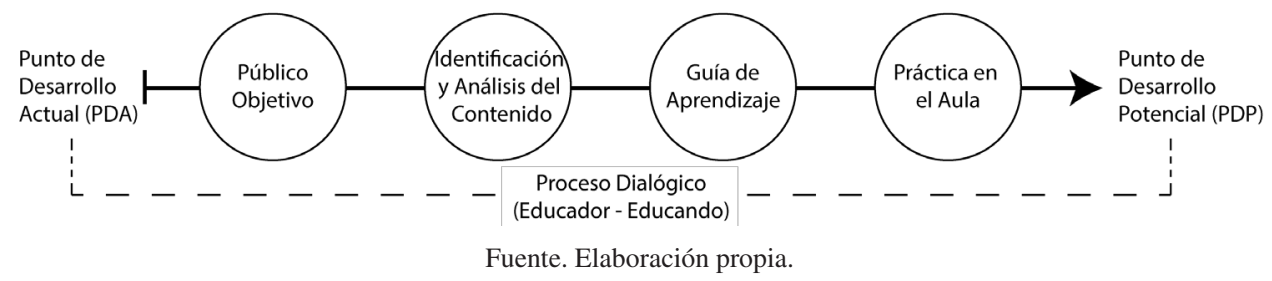

Este modelo inicia desde un Punto de Desarrollo Actual (PDA), donde el profesor tiene un diagnóstico de las necesidades que tienen los estudiantes dentro y fuera del aula para promover en ellos un cambio social, esto incluye el comportamiento, la promoción de valores y la relación con el sector externo, para el caso de Guillermina y Candelario, con el medio ambiente. Es de esta manera como el educador diseña su objetivo de enseñanza, respondiendo a la siguiente pregunta: ¿qué cambio quiero promover en mis estudiantes? En esta etapa, también se evalúa la infraestructura física y tecnológica con la que cuenta la institución y de la que se valdrá el profesor para cumplir su objetivo.

Luego, el conocimiento del público objetivo por parte del educador es fundamental para continuar con el proceso de construcción del aprendizaje, tal como lo propone el profesor Cardona. Esto consiste en conocer las satisfacciones y necesidades del público objetivo en cuanto a los contenidos de entretenimiento, ya que, como se observó en uno de los grupos focales, los menores, pertenecientes al grupo de 9 a 12 años, no mostraron mayor interés en los capítulos visualizados.

La siguiente actividad consiste en identificar y analizar los contenidos audiovisuales o multimediales que contribuyan al logro del objetivo de enseñanza. Es importante diseñar una ficha de análisis que considere algunas características como el idioma, la duración, la proximidad cultural, las unidades temáticas, los personajes, los valores y contravalores y las emociones presentes en la narrativa. Para este caso, vale la pena agregar que los niños de 7 y 8 años están concentrados en un contenido multimedial educativo por un lapso máximo de 12 minutos antes de desviar su atención hacia otra actividad (Barreto et al., 2017). A continuación, se podrían correlacionar las unidades temáticas estipuladas en los microcurrículos con la información obtenida en las fichas y así, elaborar una guía

15 Entrevista personal, Jorge Cardona, febrero 15 de 2018. 
para la clase. Pero antes, conservando el proceso dialógico entre las partes, el docente debería realizar una prueba piloto tal y como se hizo en esta investigación, concluyendo si el contenido seleccionado es el adecuado o no.

Es ideal que la guía de aprendizaje incluya el objetivo de enseñanza, las necesidades del espacio físico y los equipos tecnológicos, las estrategias pedagógicas que describan el paso a paso de cada una y un cronograma de trabajo detallado con los tiempos, las actividades y los responsables. En esta herramienta, se contempla la evaluación y autoevaluación de los resultados del proceso desarrollado hasta ese momento con el fin de ratificar los postulados de la guía o, por el contrario, tomar decisiones en cuanto a eventuales imprevistos que puedan surgir.

Finalmente, todo se lleva a la práctica en el aula de clase hasta alcanzar un Punto de Desarrollo Potencial (PDP), es decir, al momento donde se puede evidenciar el logro del objetivo propuesto, ya que

El aprendizaje ocurre cuando un maestro, un padre, una herramienta o un compañero guía a un estudiante desde un punto de desarrollo actual hasta un punto de desarrollo potencial, [donde] cada uno sirve como mediador para facilitar la apreciación de los estudiantes en una actividad determinada (Egenfeldt-Nielsen, 2006, p. 199).

Por lo tanto, este modelo se fundamenta en un proceso dialógico permanente entre el educador y el educando, en el cual, no solo se obtiene una retroalimentación que no es posible desarrollar solo con un contenido en una pantalla, sino que también, facilita la persuasión del docente hacia el estudiante y, por ende, en el logro del objetivo de enseñanza a través de una estrategia de Edu-Entretenimiento de primera generación.

\section{DISCUSIÓN Y CONCLUSIONES}

Los episodios analizados de Guillermina y Candelario, dan señales de que la serie infantil animada contribuye y fomenta el Edu-Entretenimiento a través de los personajes y su estructura narrativa. No obstante, para lograrlo de una manera efectiva, se requiere de un proceso dialógico permanente entre el educador y el educando para que emerjan procesos de construcción de sentido, siempre, con la idea de captar la atención de los menores y fomentar procesos de enseñanza-aprendizaje efectivos y, por qué no, propiciar un cambio social en sus actitudes (Tufte, 2015). Un aspecto clave es el acompañamiento pedagógico que brinda el docente en el aula, en especial, en la formación de valores, la protección y el conocimiento de la naturaleza y la diversidad cultural de una de las regiones de Colombia, en este caso, la región Pacífico.

Con respecto a lo anterior, se evidencia la importancia de que los creadores audiovisuales o multimediales incluyan en las estrategias de Edu-Entretenimiento los valores y antivalores al momento de diseñar las historias, ya que son fundamentales para que los profesores identifiquen la esencia de los contenidos y los consideren en sus estrategias de enseñanza (Álvarez Rodríguez, 2007). Además, es interesante acotar que, del análisis de los datos obtenidos de las enseñanzas que dejan los guiones de Guillermina y Candelario, surgieron la amistad, el dolor corporal y el liderazgo como otros valores emergentes a considerar dentro de la clasificación del autor antes citado. 
De igual manera, en los grupos focales se evidenció la conexión entre los participantes de 4 a 7 años y los personajes del contenido infantil, porque son niños y niñas que al igual que Guillermina y Candelario, juegan, se divierten, aprenden, lloran, sonríen y se enojan, como ocurrió en la escena donde el abuelo se burló de Guillermina, algo que no gustó a una de las niñas participantes en los grupos focales. Como consecuencia de esto, se concluye que la audiencia que hizo parte de este estudio, se siente identificada con lo que ve en la pantalla al contagiarse por los sentimientos de los personajes, tal y como lo proponen Singhal y Rogers (2002).

Como resultado de lo anterior y para contribuir en las investigaciones teóricas del EduEntretenimiento que toman el papel de las emociones más en serio, se propone el Modelo de construcción del aprendizaje en el aula a partir del Edu-Entretenimiento, el cual se deriva de la estrecha relación entre la educación y la tecnología, de la producción de contenidos audiovisuales y digitales que tengan como propósito fomentar la estrategia de educar y entretener y del interés de los educadores por incluir estos contenidos en sus planes de aula. Este modelo pedagógico se diseña a pesar de la limitación en cuanto al número de capítulos analizados en este artículo, al número de entrevistas desarrolladas y a la no aplicación de grupos focales en la región donde transcurren las historias. No obstante, se traza un camino para reproducirlo por los docentes en el aula que tengan la necesidad y el propósito de generar cambios sociales en sus grupos de estudiantes y construir escenarios para fomentar los valores como lo menciona Feng (2016).

Para futuras investigaciones en este tipo de temas, se invita a los académicos interesados en este tema a implementar el modelo con otros contenidos, en otras culturas, e inclusive, otros contextos económicos urbanos en situación de pobreza (Armella, 2016) para saber si efectivamente el programa Guillermina y Candelario propicia situaciones de cambio en la audiencia. También, desde la persuasión narrativa con un enfoque cuantitativo, se pueden analizar los efectos de las narraciones, en cuanto a las actitudes, creencias, intención de conducta y comportamientos (Igartua, 2007; van Krieken, Hoeken \& Sanders, 2017) de los menores en los espacios académicos.

En conclusión, se propone que a partir del programa de televisión infantil, Guillermina y Candelario, que opera como un mensaje mediador de entretenimiento y aprendizaje, y las instituciones educativas y sus educadores, que cumplan con el rol de agentes de cambio, planteen estrategias de Edu-Entretenimiento de primera generación (Tufte, 2015), desde las cuales se promueva el cambio social, se complemente la falta de información en aspectos ecológicos, sociales y éticos, se persuada a los infantes para que mejoren el comportamiento individual y se eduquen sobre las normas sociales.

\section{REFERENCIAS BIBLIOGRÁFICAS}

Alhumaidan, H., Pui, K., Lo, Y. \& Selby, A. (2018). Co-designing with children a collaborative augmented reality book based on a primary school textbook. International Journal of ChildComputer Interaction, 15, 24-36. https://doi.org/10.1016/j.ijcci.2017.11.005.

Alvarez Rodríguez, J. (2007). Test axiológico. Un instrumento para detectar valores. Revista Portuguesa de Pedagogía, 41(1), 157-177.

Armella, J. (2016). Hacer docencia en tiempos digitales: Un estudio socio-pedagógico en escuelas secundarias públicas emplazadas en contextos de pobreza urbana. Estudios Pedagógicos (Valdivia), 42(3), 49-67. https://doi.org/10.4067/S0718-07052016000400003. 
Estudios Pedagógicos XLV, $\mathrm{N}^{\circ}$ 3: 127-143, 2019

CONSTRUCCIÓN DE APRENDIZAJE EN EL AULA: UNA PROPUESTA PEDAGÓGICA QUE EMERGE DEL ANÁLISIS DEL PROGRAMA DE TELEVISIÓN INFANTIL GUILLERMINA Y CANDELARIO

Barreto, D., Vasconcelos, L. \& Orey, M. (2017). Motivation and learning engament through playing math video games. Malaysian Journal of Learning and Instruction, 14(2), 1-21.

ComKids. (2015). Guillermina y Candelario en Brasil. Retrieved May 31, 2018, from http://comkids. com.br/es/guilhermina-e-candelario-no-brasil/

De Souza, K. I. \& do Amaral, S. F. (2008). El uso del vídeo digital en clase de enseñanza: Una propuesta pedagógica. Comunicar, 16(31), 457-461. https://doi.org/10.3916/c31-2008-03-035.

Del Río, P. (2000). No me chilles que no te veo. Atención y fragmentación audiovisual. Cultura y Educación, 12(4), 51-80. https://doi.org/10.1174/113564000753837043.

Egenfeldt-Nielsen, S. (2006). Overview of research on the educational use of video games. Digital Kompetanse, 1, 184-213.

Fabbro, G. \& Sánchez-Labella Martín, I. (2016). Infancia, dibujos animados y televisión pública. La difusión de valores y contravalores en la producción española y argentina. Revista Mediterránea de Comunicación, 7(1), 11-29. https://doi.org/10.14198/MEDCOM2016.7.1.1

Feng, D. (2016). Promoting moral values through entertainment: a social semiotic analysis of the Spring Festival Gala on China Central Television. Critical Arts, 30(1), 87-101. https://doi.org/1 0.1080/02560046.2016.1164387.

Fosfenos Media. (n.d.). Producción infantil, Guillermina y Candelario. Retrieved May 28, 2018, from https://www.fosfenosmedia.com/index.php/produccion-infantil/guillermina-y-candelario.

Hill, J. R., \& Hannafin, M. J. (2001). Teaching and Learning in Digital Environments: The Resurgence of Resource-Based Learning. ETR\&D, 49(3), 37-52.

Hunting, K., Grumbein, A., Cahill, M., Grumbein, A. \& Cahill, M. (2018). Watch and Learn: Gendered Discrepancies in Educational Messages on Television Channels Targeted at Boys versus Girls. Mass Communication and Society, 21(1), 115-141. https://doi.org/10.1080/15205436.2017.1370114.

Igartua, J. J. (2007). Persuasión narrativa. España: Editorial Club Universitario.

Jarvin, L. (2015). Edutainment, Games, and the Future of Education in a DigitalWorld. New Directions for Child and Adolescent Development, (147), 33-40. https://doi.org/10.1002/cad.20082.

La Ferrara, E. (2016). Mass media and social change: can we use television to fight poverty? Journal of the European Economic Association, 14(4), 791-827. https://doi.org/10.1111/jeea.12181.

Lindlof, T. \& Taylor, B. (2002). Qualitative Communication Research Methods. Thousand Oaks, London \& New Delhi: Sage Publications.

López Neira, L. R. (2017). Indagación en la relación entre aprendizaje - tecnologías digitales. Educación y Educadores, 20(1), 89-105. https://doi.org/10.5294/edu.2017.20.1.5

MaguaRed. (2015). Maguared. Cultura y primera infancia en la web. Retrieved May 18, 2018, from https://maguared.gov.co/project/guillermina-y-candelario/

Perceval, J. M. \& Tejedor, S. (2008). Oral-gestual, escritura, audio, audiovisual, y idigital? Los cinco grados de la comunicación en educación. Comunicar, 15(30), 155-163. https://doi.org/10.3916/ c30-2008-02-009.

Rincón, M., Rincón, M. \& de Jesús, U. (2010). Guillermina y Candelario. Colombia: Señal Colombia.

Rivera, P. (2017). Mi Señal de Señal Colombia, ahora mejor que nunca. Retrieved June 1, 2018, from http://aprende.colombiaaprende.edu.co/es/agenda/noticias/mi-señal-de-señal-colombia-ahoramejor-que-nunca.

RTVC. (2016). Señal Colombia. Guillermina y Candelario. Retrieved May 18, 2018, from http:// www.senalcolombia.tv/programas/guillermina-y-candelario.

Señal Colombia. (2017). Llega una nueva temporada de Guillermina y Candelario a Mi Señal. Retrieved September 8, 2014, from https://www.misenal.tv/noticias/para-grandes/guillerminay-candelario/llega-una-nueva-temporada-de-guillermina-y-candelario.

Singhal, A. \& Rogers, E. M. (2002). A Theoretical Agenda for Entertainment- Education. Communication Theory, 12, 117-135. https://doi.org/10.1111/j.1468-2885.2002.tb00262.x

Tufte, T. (2001). Entertainment-education and participation. Journal of International Communication, 7(2), 25-50. https://doi.org/10.1080/13216597.2001.9751908. 
. (2015). Comunicación para el cambio social. La participación y el empoderamiento como base para el desarrollo mundial. Barcelona: Icaria Editorial.

van Krieken, K., Hoeken, H. \& Sanders, J. (2017). Evoking and measuring identification with narrative characters - A linguistic cues framework. Frontiers in Psychology, 8, 1-16. https://doi. org/10.3389/fpsyg.2017.01190.

Zabala, J. D. (2016). Reflexividad, mediaciones y educación. El sujeto y su interacción con la pantalla audiovisual. Revista Colombiana de Ciencias Sociales, 7(2), 349-369. https://doi. org/10.21501/22161201.1731. 
